\title{
A MIXED BOUNDARY VALUE PROBLEM SOME REMARKS ON A PROBLEM OF A. WEINSTEIN
}

\author{
ALBERT E. HEINS
}

At present the unilateral Laplace transform has had many interesting applications. To cite several types we have (a) initial value problems in ordinary differential equations, (b) initial value and boundary value problems in partial differential equations with one space variable, (c) "initial value problems" in ordinary difference equations, and (d) "initial value" and "boundary value problems" in partial difference equations.

Titchmarsh ${ }^{1}$ and his collaborators, Cooper ${ }^{2}$ and Busbridge, ${ }^{3}$ have indicated that much can be done with the finite Laplace transform, that is,

$$
\int_{a}^{b} e^{-s x} f(x) d x=g(s) .
$$

The transform (1) contains as a special case a finite Fourier transform, first used by Stokes in 1850 for the solution of certain boundary value problems in mathematical physics. The finite Fourier transform has been recently revived by Doetsch ${ }^{4}$ and Kniess. ${ }^{5}$ It cannot be applied as widely as (1), since it assumes, a priori, that the boundary value problem naturally has a Fourier series as an expansion. On the other hand (1) slips quite neatly into an expansion which is natural to the boundary value problem.

By applying a transformation of the type (1) to a linear differential equation (ordinary or partial) with constant coefficients under given boundary conditions, boundary functions which are superfluous are introduced. Picone and others have demonstrated that by solving the reduced equation and noting the fact that $g(s)$ is an entire function of the parameter $s$, these superfluous boundary elements may be eliminated. This procedure may become exceedingly difficult to carry through. The method we employ here makes use of a regularity condition which is introduced by rendering the boundary conditions symmetric.

Received by the editors April 25, 1942.

1 Titchmarsh, J. London Math. Soc. vol. 14 (1939) p. 118.

${ }^{2}$ Cooper, J. London Math. Soc. vol. 14 (1939) p. 124.

${ }^{3}$ Busbridge, J. London Math. Soc. vol. 14 (1939) p. 128.

${ }^{4}$ Doetsch, Math. Ann. vol. 112 (1935) p. 52.

${ }^{5}$ Kniess, Math. Zeit. vol. 44 (1939) p. 266. 
We propose to treat here a mixed boundary value problem in potential theory with the aid of (1). This problem was first investigated by A. Weinstein in 1927 and later by Cooper, ${ }^{6}$ Bochner and Poritsky. The author wishes to thank Dr. Alexandre Weinstein for having brought this problem to his attention and for having discussed it with him.

We are concerned here with the following problem. Find the conditions under which the solution of the harmonic equation

$$
\frac{\partial^{2} u}{\partial x^{2}}+\frac{\partial^{2} u}{\partial y^{2}}=0
$$

under the mixed boundary conditions

$$
u(x, 0)=0, \quad u(x, 1)=k u_{y}(x, 1)
$$

may be bounded or of finite exponential order for $-\infty<x<\infty$. We modify this problem by considering the solution of (2) which is odd and continuous in $y$ and which satisfies the mixed boundary conditions

$$
u(x, 1)=k u_{y}(x, 1), \quad-u(x,-1)=k u_{y}(x,-1) .
$$

We write

$$
g(x, s)=\int_{-1}^{1} e^{-s y} u(x, y) d y .
$$

Since $u(x, y)$ is a harmonic function we may differentiate twice under the integral sign with respect to $x$, and we have

$$
\frac{\partial^{2} g}{\partial x^{2}}=-\int_{-1}^{1} e^{-s y} \frac{\partial^{2} u}{\partial y^{2}} d y .
$$

Upon integrating by parts and noting the boundary conditions of the modified problem we have

$$
\begin{aligned}
\frac{\partial^{2} g}{\partial x^{2}}+s^{2} g & =-e^{-s} u_{y}(x, 1)+e^{s} u_{y}(x,-1)-s\left[e^{-s} u(x, 1)-e^{-s} u(x,-1)\right] \\
& =u_{y}(x, 1)\left[\left(e^{s}-e^{-s}\right)-s k\left(e^{s}+e^{-s}\right)\right] .
\end{aligned}
$$

But

$$
u_{y}(x, 1)=\frac{\left(\partial^{2} g / \partial x^{2}\right)+s^{2} g}{e^{s}-e^{-s}-s k\left(e^{s}+e^{-s}\right)}
$$

${ }^{6}$ Loc. cit. 
and (3) is finite for all $s$. Hence

$$
g_{x x}\left(s_{j}\right)+s_{j}^{2} g\left(s_{j}\right)=0
$$

for all $s_{j}$ which are roots of

$$
e^{s}-e^{-s}-s k\left(e^{s}+e^{-s}\right)=0 .
$$

Hence

$$
g\left(x, s_{j}\right)=A\left(s_{j}\right) e^{i s_{j} x}+B\left(s_{j}\right) e^{-i s_{j} x}=\int_{-1}^{1} e^{-s_{j} y} u(x, y) d y .
$$

Now if $k>1,{ }^{7}(4)$ has a real root at $s=0$ and an infinite sequence of conjugate imaginary roots. If $k<1,(4)$ has three real roots and an infinite sequence of conjugate imaginary roots. As a matter of notation let $s_{j}=i \alpha_{j}\left(i=(-1)^{1 / 2}\right)$. If $u(x, y)$ is to be bounded for all $x, s_{j}$ is real and hence all $A\left(s_{j}\right)$ and $B\left(s_{j}\right)$ are zero save for the coefficients $A$ and $B$ corresponding to the real $s_{j}$. If $u(x, y)$ is to be of finite exponential order, say $O\left(e^{\lambda x}\right)$, then $\left|s_{j}\right|<\lambda$ and all $A\left(s_{j}\right)$ and $B\left(s_{j}\right)$ are zero for any $\left|s_{j}\right|$ greater than $|\lambda|$. Thus without going to the expense of solving a nonhomogeneous differential equation and evaluating complex integrals as Cooper did, we can get the same results he did by elementary methods.

We can of course obtain Cooper's expansion by noting that the set of functions $\left\{\sin \alpha_{j} y\right\}\left(i s_{j}=\alpha_{j}\right)$ is a complete set of orthogonal functions over $-1 \leqq y \leqq 1$. If we then assume that $u(x, y)$ is of bounded variation with respect to $y$ in $-1 \leqq y \leqq 1$ (or any other such condition which will insure an adequate representation) we have

$$
\sum_{j=-\infty}^{\infty} C_{j} e^{i \alpha_{j} y} \int_{-1}^{1} e^{-i \alpha_{j} y^{\prime}} u\left(x, y^{\prime}\right) d y^{\prime}=u(x, y)
$$

where the $C_{j}$ 's are the normalizing factors of the set $\left\{\sin \alpha_{j} y\right\}$. Then if $u(x, y)$ is bounded with respect to $x,(5)$ appears as a sum of two terms (that is, the case $k>1$ ). If $u(x, y)$ is of finite exponential order with respect to $x,(5)$ appears as a finite sum. It is to be noted that since no conditions other than order conditions were put on $x$, for large $x$, the $A$ 's and $B$ 's remain undetermined.

The technic we have employed can be applied to the solution of Laplace's equation in two or three variables, when the boundary of the domain over which we solve this equation is a rectangle. The same technic may be applied to the solution of characteristic value

${ }^{7}$ The critical case $k=1$, follows as the discussion in the text. In this case a first degree polynomial in $x$ arises instead of the exponential functions. 
problems associated with the differential equation

$$
\frac{\partial^{2} u}{\partial x^{2}}+\frac{\partial^{2} u}{\partial y^{2}}+\lambda u=0
$$

for various types of boundary conditions when the boundary is rectangular.

Purdue University

\section{ON THE CONVERGENCE OF A CONTINUED FRACTION}

\section{T. F. GLASS AND WALTER LEIGHTON}

It is known [1] that sufficient conditions for the convergence of the continued fraction

$$
b_{0}+\frac{a_{1}}{1}+\frac{a_{2}}{1}+\cdots
$$

where the elements are complex numbers, are

$$
\left|a_{2}\right| \geqq 5, \quad\left|a_{2 n}\right| \geqq 25 / 4, \quad\left|a_{2 n-1}\right| \leqq 1 / 4, \quad n=2,3,4, \cdots .
$$

The purpose of this note is to extend this result.

THEOREM. If $\left|a_{2 n+1}\right| \leqq r \leqq 1 / 4(n=1,2,3, \cdots)$ and if the numbers $a_{2 n}=\rho_{2 n} e^{i \theta_{2 n}}(n=1,2,3, \cdots)$ satisfy the conditions

$$
\begin{array}{lrl}
\rho_{2 n} & \geqq 2(1+r)^{2}\left[1-\cos \left(\theta_{2 n}+\theta_{0}\right)\right], & 0 \leqq \theta_{2 n}<\pi-\theta_{0}, \\
\rho_{2 n} \geqq 4(1+r)^{2}, & \pi-\theta_{0} \leqq \theta_{2 n} \leqq \pi+\theta_{0}, \\
\rho_{2 n} \geqq 2(1+r)^{2}\left[1-\cos \left(\theta_{2 n}-\theta_{0}\right)\right], & \pi+\theta_{0}<\theta_{2 n} \leqq 2 \pi,
\end{array}
$$

where $\theta_{0}=2$ arc $\sin r$, the continued fraction (1) converges.

To prove the theorem we employ the continued fraction

$$
1+\frac{x_{1}}{1}+\frac{x_{2}}{1}+\cdots
$$

where

$$
x_{2 n}=\frac{\left(1+a_{2 n-1}\right)\left(1+a_{2 n+1}\right)}{a_{2 n}}, \quad n=2,3, \cdots
$$

Presented to the Society, September 5, 1941; received by the editors May 7, 1942. 